\title{
Toward Designing Information Display to Support Critical Care
}

\section{A Qualitative Contextual Evaluation and Visioning Effort}

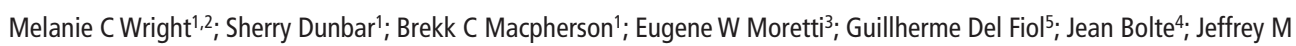
Taekman ${ }^{3}$; Noa Segall ${ }^{3}$

${ }^{1}$ Saint Alphonsus Health, Boise ID; ${ }^{2}$ Trinity Health, Livonia MI; ${ }^{3}$ Duke University Medical Center, Department of Anesthesiology, Durham, NC; ${ }^{4}$ Duke Clinical and Translational Science Institute, Durham, NC; ${ }^{5}$ University of Utah Department of Biomedical Informatics, Salt Lake City, UT

\section{Keywords}

Electronic health records and systems, clinical decision support, monitoring and surveillance, clinical documentation and communications, intensive and critical care, human-computer interaction, interfaces and usability, safety, qualitative

\section{Summary}

Objectives: Electronic health information overload makes it difficult for providers to quickly find and interpret information to support care decisions. The purpose of this study was to better understand how clinicians use information in critical care to support the design of improved presentation of electronic health information.

Methods: We conducted a contextual analysis and visioning project. We used an eye-tracker to record 20 clinicians' information use activities in critical care settings. We played video recordings back to clinicians in retrospective cued interviews and queried: 1) context and goals of information use, 2) impacts of current display design on use, and 3) processes related to information use. We analyzed interview transcripts using grounded theory-based content analysis techniques and identified emerging themes. From these, we conducted a visioning activity with a team of subject matter experts and identified key areas for focus of design and research for future display designs.

Results: Analyses revealed four unique critical care information use activities including new patient assessment, known patient status review, specific directed information seeking, and review and prioritization of multiple patients. Emerging themes were primarily related to a need for better representation of dynamic data such as vital signs and laboratory results, usability issues associated with reducing cognitive load and supporting efficient interaction, and processes for managing information. Visions for the future included designs that: 1) provide rapid access to new information, 2) organize by systems or problems as well as by current versus historical patient data, and 3) apply intelligence toward detecting and representing change and urgency.

Conclusions: The results from this study can be used to guide the design of future acute care electronic health information display. Additional research and collaboration is needed to refine and implement intelligent graphical user interfaces to improve clinical information organization and prioritization to support care decisions. 


\section{Correspondence to:}

Melanie C. Wright, PhD

Program Director, Patient Safety Research Trinity Health and Saint Alphonsus Health System 1055 N. Curtis Rd

Boise ID 83702

Email: melanie.wright@trinity-health.org Phone: 208-367-7399
Appl Clin Inform 2016; 7: 912-929

http://dx.doi.org/10.4338/ACl-2016-03-RA-0033

received: March 15, 2016

accepted: August 23, 2016

published: October 5, 2016

Citation: Wright M, Dunbar S, Macpherson B, Moretti EW, Del Fiol G, Bolte J, Taekman JM, Segall, N. Toward designing information display to support critical care: A qualitative contextual evaluation and visioning effort. Appl Clin Inform 2016; 7: 912-929

http://dx.doi.org/10.4338/ACI-2016-03-RA-0033

Funding

This project has been funded with Federal funds from the Department of Health and Human Services, National Institutes of Health, National Library of Medicine under Grants \#R21 LM010700 with Duke University and \#R56 LM011925 with Saint Alphonsus Regional Medical Center and the Agency for Healthcare Research and Quality Grant \#K02 HS015704. 


\section{Background and Significance}

Each year, tens of thousands of patients are harmed because important patient information is missed, misunderstood, or identified too late $[1,2]$. Critical care settings, in particular, are vulnerable to problems related to failures in accessing and interpreting critical diagnostic information [3-7]. Critical care patients can receive as many as 178 interventions daily [3]. Data relevant to care decisions and management are complex, and come from a variety of electronic, paper, and verbal sources [3]. It is increasingly difficult for providers to search through these data and locate information important to care decisions. Inefficiencies and the opportunity to miss key information make electronic health care information presentation a crucial patient safety priority [8].

Electronic health records (EHRs) and other health care information technology (IT) that provide patient health information have been widely linked to usability problems [9-13]. Physicians identified EHRs as one of the primary contributors to professional dissatisfaction [14]. Among issues like time-consuming data entry and poor clinical documentation quality, physicians identified user interfaces that do not match clinical workflow and information overload as key problems. Veteran's Administration EHR users describe usability problems related to locating desired information, presentation of information, and prioritization of data $[15,16]$. A recent review by Zahabi, Kaber, and Swangnetr identified 46 articles that focused on usability and user interface design in the context of EHRs [13]. From these articles, they summarized 65 guidelines (eg, "Cluster related information on the same screen [17]") toward the design of more usable EHRs [13]. According to the American Medical Informatics Association EHR 2020 Task Force on the Status and Future Direction of EHRs, there is a need to improve information design so that EHRs support and build upon how people think [18].

Research studies have explored issues of usability, clinician information needs, and novel design approaches to information presentation in the critical care setting. Carayon et al used direct observation to evaluate the impact of EHRs on time spent in different high-level categories of clinical tasks [19]. Other researchers have compared different approaches for organizing patient information [20,21], presenting vital signs and other bedside data [22-24], presenting problem lists [25], or presenting laboratory data $[24,26]$. Researchers have evaluated the use of computer surveillance to support identification and presentation of information around specific clinical problems, such as hospital-acquired infections [27] or ventilator-induced lung injury [28]. Research has also explored information use behaviors and information needs [29-32]. Pickering et al applied these findings to the design of a novel critical care information system organized by human body systems [20].

While usability design guidelines provide general guidance for the presentation of electronic health information, and research in the critical care setting has begun to explore new information presentation approaches for this data rich environment, there remains a need for a broader understanding of the goals and contexts of critical care information use. Additional information is needed to understand how critical care clinicians use information and how best to organize and prioritize information to support their needs. There is a need to explore how different approaches, such as the application of computer intelligence or advanced graphical information presentation techniques, may be applied to solve problems of data overload and support clinician decision-making.

In user-centered design approaches, systems are designed around the needs and capabilities of users. This typically begins with an in-depth study of the work environment and the needs of the intended end users [33-37]. An iterative process of design and evaluation with representative users then follows, progressing from design concepts to increasingly detailed designs and prototypes. While quantitative approaches to understanding the user and work environment may classify and quantify use behaviors in a general way, qualitative interpretation by clinicians, such as a description of what they are doing and why, is important for generating recommendations for improvement. Outside of the care setting, researchers have used surveys, interviews and focus groups to gather qualitative information $[14,15,33]$. However, problems of recall and bias may limit the range and validity of data collected outside of the work context $[38,39]$. Contextual field inquiry involves observations and interviews conducted in real-time during work activities [34], but is disruptive in busy clinical settings. Combining field observations with outside of care setting interviews is one alternative $[31,32,40]$. Opportunistic interviewing, asking questions in the clinical setting when op- 
portunities arise, is another alternative that supports capture of contextually grounded qualitative data [31].

Eye-trackers have been used for healthcare research both in simulated settings and in the field [41-46]. Analysis of data can be qualitative or quantitative (eg, the number of times or amount of time participants fixate on specific information, number of transitions between specific information elements). Encoding of eye-tracking fixation data for quantitative analysis is difficult for a number of reasons [47-49]. The technology requires calibration by individual and there are frequent technological problems and high rates of data loss. If expected eye-gaze positions can be pre-defined and fixed in location, complex software can be used to automate fixation data collection. In naturalistic settings, this is usually difficult, if not impossible, and encoding of fixation data must be completed manually (with a person watching frame by frame and documenting the information viewed). For these reasons, quantitative analysis of naturalistic eye-tracking data is usually not feasible except to evaluate a specific question about information access [44].

While quantitative analysis of eye-tracking data in naturalistic settings is difficult, qualitative analysis of eye-tracking data provides a good alternative for combining the rich contextual data provided through observation and rich qualitative data provided through interviews. Using this method, clinician information use behaviors, including visual attention using an eye-tracker, are recorded and the recording replayed for clinicians, away from the care setting, asking them to describe and rationalize their behaviors $[44,50]$. Compared to opportunistic interviewing, this approach supports greater rigor with respect to controlling times and settings of data capture, while still allowing for in-depth queries regarding motivations and rationale. Participants also are better able to reflect on their motivation and work processes when they are not simultaneously trying to complete their normal clinical work $[43,44]$.

\section{Objectives}

Our primary objective was to better understand the way critical care clinicians use information to make care decisions. We sought to supplement previous research in critical care $[6,19,30,32]$ with rigorous in-depth qualitative analysis of naturalistic behaviors during times of heavy information use. From these findings, we sought to identify opportunities for improvements in the design of critical care electronic health information displays. In particular, we were focused on identifying priorities and opportunities with respect to application of computer intelligence or advanced graphical information presentation techniques.

\section{Methods}

\subsection{Overview}

In this article, the term participant refers to clinician-participants in eye-tracking and interview activities. The term provider refers to physicians (including residents and fellows) and nurse practitioners (NPs) and the term clinician refers to providers and nurses. Our approach involved methods appropriate for early phases of user-centered design focused on (1) understanding the information needs and work context of the users and, based on these, (2) iterative visioning of opportunities for improving information presentation. First, we used a head-mounted eye-tracker to gather video data, audio data, and the clinician's eye position during information use activities including use of both electronic and other information sources such as paper or other clinicians [46]. We replayed the recording as context for clinician interviews away from the care setting $[44,50]$. To analyze the data, we applied grounded theory-based content analysis techniques to transcribed interview data [51]. We then analyzed the interview outcomes with a small group of providers and designers in an iterative process to explore opportunities for innovation (visioning). 


\subsection{Eye tracking care settings and participants}

We recorded information use activities and interviewed 20 participants on three critical care units at two hospitals with different information systems and practice models (a large academic hospital and a community hospital). Settings included the Duke University Hospital surgical ICU (SICU), neurological ICU (NICU), and the Saint Alphonsus Regional Medical Center (SARMC) intensive care unit (ICU). At the time, Duke University used a combination of commercially available and internally developed healthcare IT and SARMC used a large commercially available integrated EHR. Participants included physicians, residents, fellows, nurse practitioners (NPs), and critical care nurses, covering the range of clinicians involved in care decisions ( Table 1). We worked with participants to identify time periods when information use was high. These included group rounding (periodic team-based patient evaluation including data review and physical assessment), individual rounding (periodic patient review by a single clinician), reviewing patient data before rounding, sign outs (care transfer), and patient admissions.

\subsection{Eye tracking data capture and interview}

After recruiting a participant and identifying the time period for data collection, we obtained informed consent, placed an ASL Mobile-Eye mobile glasses-mounted eye tracker on the participant, and recorded the wearer's field of view (video), audio, and pupil position for one hour while they completed their normal clinical tasks ( Figure 1). The number and type of patients' data captured depended on clinician type and activities during recording. For example, information use by nurses generally focused on one or two patients, while providers usually accessed data for multiple patients including detailed data on one or two patients during the hour of data capture. For privacy reasons, we did not record inside patient rooms. Thus, we did not directly capture use of information from patient physical assessment or bedside monitor data. However, interview discussion included the use of this type of information.

After recording, we created a video (and audio) playback that displayed a red cursor to represent eye position. Within one to two days after data collection, we conducted a retrospective verbal protocol interview $[44,50]$. The interviews lasted from 60 to 90 minutes. The interviewers first queried participants about their roles and responsibilities on the day the data were recorded, and then played the video recording. While viewing the video, participants were asked to describe:

1. the type of information they were accessing and why;

2. their use of paper, electronic, and other information sources; and

3. the situation that was recorded and how it translated to other patients or contexts.

Interviewers also used recorded situations as a starting point for additional discussion about information use. For example, when watching playback of documentation of information on paper, the interviewer asked the participant to describe not only that specific activity, but also to describe their use of paper in general, and how the information would be used later. The interview was audio-recorded and transcribed for analysis.

\subsection{Data coding, analysis, and visioning}

Because our primary objective was to understand the way critical care clinicians use information to support care decisions, we used contextual design interview interpretation methods [52] targeted toward understanding goals and contexts of clinician information use. Specifically, we quantified and classified:

1. relevant triggers or cues to seek out data, such as preparing for rounds;

2. sources of data used - paper, electronic, or verbal;

3. goals such as making a decision about patient transfer; and

4. characteristics of data engagement such as lookup of an anticipated lab value.

From these, we classified common information use contexts, goals, and the relevant information associated with each. 
We were also interested in exploratory analysis of the data, without predefined themes for investigation. Using grounded theory methods, we conducted a first pass through our data using open coding to group incidents or issues with similarities into themes which are named according to meaning [51]. Using simultaneous data collection and analysis, early interviews informed the selection of individuals, settings, time periods, and queries for later data collection. We renamed, reorganized, and redefined emerging themes through an ongoing process of refinement. We used axial coding to explore and define relationships between categories. Two PhD trained and one master's trained human factors professionals coded the data. Every interview was coded by at least two coders and all three worked together to construct emerging themes and generate memos on themes. In addition to a brief discussion of each topic, memos listed relevant codes and direct quotes from participants.

After coders agreed they were attaining no new information from the eye-tracking interviews and coding, we summarized the findings of both the information use contextual analysis and grounded theory emerging themes. We validated the findings with five participants - one physician and one nurse practitioner involved in the eye-tracking interviews, two additional critical care physicians, and one physician-informaticist. Participants were asked specifically whether they disagreed with any of the findings.

In order to translate the findings from our analysis into actionable ideas to apply intelligence and other innovations to improve information presentation in critical care, we then conducted a visioning activity. The visioning team included the same eight individuals involved in coding and validating the eye-tracking interviews. The team provided expertise in human factors, design, biomedical informatics, critical care, and process and technology innovation in healthcare. Through several consensus meetings, the three human factors designers combined information from the memos and themes with their background knowledge of informatics and user interface design literature and experience with a variety of user interface design applications into an initial summary and visioning discussion document (supplemenatry file Appendix A). This was used as a framework for iterative visioning meetings with the remaining team members.

Each team member reviewed the initial visioning document and, in the context of recorded meetings, commented on and added to innovation ideas related to information presentation. Ideas raised by one individual were raised and discussed in subsequent meetings. Meeting discussions were transcribed and coded. Coding of meeting transcripts involved documenting specific innovation ideas with support from multiple team members for inclusion in our findings. Initial stages of the visioning process involved primarily text descriptions of ideas for improvement and later stages involved the development and feedback on graphical examples of key concepts.

\subsection{Validity}

We took steps to ensure that data were reliable and comprehensive [53, 54]. Steps included:

1. collecting data from a variety of different individuals and in different settings, as described,

2. staying "close to the data" by keeping track of specific data (transcribed statements of the participants) that were associated with codes and themes,

3. collecting data to saturation [51] -- all coders agreed that the information use interviews were no longer yielding new information related to general themes of information use in the context of critical care,

4. using 3 coders who created, recorded, and followed rules regarding concepts and categories,

5. soliciting feedback from a subset of participants on data interpretations, and

6. incorporating a larger team of experts in the interpretation of interview findings into actionable design innovations.

\section{Results}

Although information creation (documentation) and information use are highly interdependent, our work was primarily centered on information use. In the discussion of our findings, we use the term dynamic data to refer to data that is updated frequently (daily or many times per day) includ- 
ing vital signs, ventilator settings and data, fluid intake and output, laboratory values, physical and nursing assessments, interventions, procedure findings, imaging, and medications. Example quotes representing the themes and additional detail on content provided by interviewees is provided in supplemenatry file $>$ Appendix A. $>$ Table 2 and $>$ Table 3 provide summaries of the findings of each of the different steps of our analyses. In the following section, we provide a description of these findings. We provide only brief descriptions of emerging themes already well described in the literature.

\subsection{Contextual design interpretation: Context and goals of information use}

Our interviews defined the role of critical care clinicians as caring for the whole patient with a focus on acute problems. Although they consider long-term goals, such as managing chronic conditions, they focus primarily on acute problems and stabilizing the patient so that they can be transferred out of critical care for further treatment: "You might have COPD, but why they're in the ICU is septic shock." Critical care providers perceived these aspects of their care activities as differentiating them and their information needs from other clinicians: "As an intensivist, our responsibility is to take care of the entire body. The urologists, they just care about the stream. Cardiologists just care about the pump. ... It's not until you get into critical care that you have to tie all those things together."

We identified 4 distinct information use activities, each with unique contexts and goals related to caring for critical care patients:

1. new patient assessment,

2. reviewing the status of a known patient,

3. specific directed information seeking, and

4. reviewing and prioritizing multiple patients.

Although we describe these as discrete activities, they represent a continuum of information use, and can be inter-related and simultaneous. Rarely does a clinician approach an information search without prior information about the patient, and without specific care goals that drive the search. Frequently, information they find will contribute to decisions to look for more information.

The goal of a new patient assessment is to establish a "big picture" understanding of the patient. It occurs when a patient is new to the unit or to the clinician. Relevant information may include: past medical history, current "story" (why they are in the ICU), current/acute and chronic problems and diagnoses, home and current medications, allergies, current and past major medical procedures, other clinicians involved (and what they report), problem-specific information (such as blood pressure stability in a hypertensive patient or insulin levels in a diabetic patient) and, if relevant, treatment plans and current stay dynamic data.

The goal of a known patient status review is to periodically review patient data to evaluate patient progress toward goals or expectations. It occurs during handovers, team rounds, when assuming care responsibility, when preparing for rounding, when preparing for a milestone (eg, transfer out or extubation), when completing documentation, and when new data are expected (eg, timing of new lab reports). The frequency and comprehensiveness of review depends on factors such as patient stability, planned interventions, clinician's familiarity with the patient, and other providers involved. Relevant information may include the full range of clinical data available from electronic or paper sources, bedside examination, or verbally from other clinicians. Clinicians are most frequently focused on dynamic data and their stability or variability over time: "The patient has septic shock, is on a ventilator, has renal failure. I look at information pertinent to those diagnoses ...seeing if she is improving ... looking at change rather than absolute."

The goal of specific directed information seeking is to review specific data to evaluate patients' response to therapy and, if relevant, adjust care plans. In some cases, speed of access to information can impact patient outcomes. Clinicians seek specific information at pre-identified times such as a period of time after a treatment has been initiated or a time when data such as new lab reports are published: "So what I was writing on my sheet was 'follow up INR' later today. Don't be fooled by the fact that you have a normal value and stop looking." Relevant information is usually a specific dy- 
namic data element. Depending on the information obtained, additional data may be sought to better understand and treat the problem.

The goal of reviewing and prioritizing multiple patients is to generate a list, or "census", of all the patients clinicians are responsible for in some way. The list is used to make sure patients aren't missed and to prioritize patients to "see" (eg, physical assessment, review information, manage therapy). While a list can, to some degree, be generated automatically by EHRs, we observed providers searching through information, such as progress notes, to determine whether a specific patient should be included on their list. This activity usually occurs at the start of a shift, but may also occur periodically throughout the day as providers identify changes, or track specific patients or interventions. Relevant information may include: patient assignment information and dynamic data or other patient data relevant to interpreting patient acuity or stability. Information may be obtained from electronic or paper systems or from verbal communication (for example, a provider may be asked by another provider to follow up on a specific patient or a provider may be told during a handover which specific patients have urgent needs).

\subsection{Emerging themes from grounded theory coding of eye-tracking in- terviews}

\subsubsection{Representing dynamic data}

Several themes that emerged from our interviews were related to clinician concerns about accessing and interpreting the large quantity of dynamic data that is generated during an ICU stay. Clinicians were firstly focused on the status of the patient at the current moment or "what's new". Even with a new patient, when historical data is required to gain a complete understanding of the patient, the primary goal is to understand and manage acute problems; and current dynamic data are usually most important. Clinicians most frequently access information for a patient they already know and are looking for the most current information to evaluate changes: "I'm just pulling up the most recent lab results, to see what we've had recently, how old they are, look at the time on them... they update these numbers anywhere from 3 am until 6 am, it can take a few hours, so it could be subsequent data that's come in since they reviewed that patient." While this was prioritized in some displays, sorting recent data to the top, frequently clinicians had to sort through or ignore data that they had seen many times in order to get to new information.

Many providers reminisced about large paper-based flow sheets that could be unfolded, and displayed vital signs, fluids in and out, drips, infusions, and sometimes, laboratory data in a graphical representation over time, generally the past 24 hours. Most clinicians were dissatisfied with the electronic presentation of dynamic quantitative data. In comparison to paper, interviewees described current systems as superior for managing historical, administrative, and text-based data, such as notes, but poor for presenting quantitative data.

Frequently, clinicians described the need to evaluate trends, changes in dynamic quantitative data over time. Increases or decreases in vital signs or lab values are used to determine whether a patient is getting better or worse, and to assess the effectiveness of different treatments. Rate of change over time can also be important. While some systems provided the ability to generate graphical presentation of quantitative data, clinicians found the steps required were cumbersome, or the processing time to load the display too long: "She's talking about changes in liver function studies. I was probably trending that data, just numbers in a row, not plotted graphically, some things you can, but I don't usually bother. It just takes too long."

Related to the need to monitor changes in quantitative data over time, clinicians also frequently described the need to see related information together. Historical flow sheets, for example, allowed clinicians to see infusions and drips, and their concomitant effect on vital signs and fluids in and out plotted on the same time scale. Seeing different quantitative data plotted together can also have important implications for diagnoses (eg, evaluate whether an increase in one metric is coincident with a decrease in another): "Take my p02 and fi02 and my PEEP which is a ventilator thing, not a laboratory thing .... and you can't do that [see them together] in this system. It's gotta be inferred and that's really hard to do..." 
Clinicians described laboratory data as vitally important to care decisions. Timing of an information search frequently depends on the availability of new laboratory data. Laboratory data are complex and voluminous. A blood chemistry screen, for example, can measure up to 19 different substances in the blood. Critical care clinicians may want to follow the trend of 3 or 4 of those values, and scan for anything aberrant in a few others. Clinicians stated that coding or highlighting low, high, or critical values provides additional information, but that critically ill patients often have many measures out of the normal range for extended periods, which minimizes the value of this technique for focusing attention.

\subsubsection{Usability Problems}

Our interviews did not focus on usability problems of specific systems; however, several usability problems that were prevalent across systems, settings, and relevant to the critical care setting emerged as priorities with respect to supporting critical care information use in our analysis. Clinicians indicated that speed of information retrieval was perhaps the most important factor when deciding where to go for information. Displays that required clinicians to wait more than a second or two to load were avoided, sometimes even if the information organization was preferred. Complexity associated with getting to important information was identified in many different contexts. With respect to system and navigation complexity, providers generally used proven paths to get to needed information, even if more efficient paths were available. Users also described that custom-filtered displays, intended to provide quick access to frequently used information for specific clinicians, fell short of their needs. While display clutter and hidden information in general were concerns, concerns about clutter were usually associated with presentation of dynamic data updated during the course of the patient's stay in the ICU. With regard to clinical decision support (CDS) systems that use patient data as criteria to "push" information, clinicians were frustrated by broad-based (presented to everyone), repetitive (lack the sophistication to allow users to turn them off or temporarily disable them when they are not relevant), and disruptive nature of pop-up windows. Participants also frequently commented on problems with cross-system integration.

\subsubsection{Processes for recalling and sharing information and for tracking tasks}

Some information searches were for the purposes of gathering and preparing information for later recall, sharing with others, and tracking task completion. This information was recorded on paper or in a different electronic form for later use. At both Duke University ICUs, NPs and residents devoted significant time to the development of summary information documents to support team activities. Clinicians also develop paper-based summary information for personal use. These practices both served as means to work around the difficulty of accessing key information in the electronic systems and as a method for developing a strong understanding of the patient situation. Details of these practices are described in supplemenatry file $>$ Appendix A.

Most clinicians perceived daily progress notes to be important information for care decisions. Clinicians commented that the quality of notes varies by provider, and expressed frustration with difficulty in finding and transferring specific quantitative dynamic data to include in the note, because it was not possible or was difficult to directly transfer the data to the note or to even see the data on the screen while writing the note. Clinicians used a variety of information sources to generate and track tasks to be completed. While some computer systems supported tracking nursing tasks, providers primarily used their own or their team's paper-based or custom systems, as well as evidence-based templates or checklists, for task tracking.

\subsection{Visioning future information display to support critical care}

In the visioning activity, our iterative process refined the 12 concepts presented as opportunities in supplemenatry file $>$ Appendix A to the eight summarized in $>$ Table 3 . Iterative meetings focused on clinician input led to three new concepts, modified three concepts, and confirmed two concepts from the initial visioning framework. In general, the team agreed that there is a pressing need for better use of graphical user interface solutions. Specific ideas to support improved organization and navigation of information and to support the situation awareness of critical care clinicians were gen- 
erated. The team supported a vision of more intelligent approaches toward organization and prioritization of information, but raised several caveats:

1. systems must be designed with a strong focus toward consistency in information presentation and user interaction [13],

2. any intelligence that is used must be transparent and comprehensible to clinicians, and

3. features with a time cost to clinicians are unlikely to be accepted.

Table 3 provides detailed descriptions of the eight areas of focus for design and research that resulted from the iterative visioning activity ( $>$ Appendix A for example quotes from team meetings). The visioning activity also led to the development of a conceptual display ( $\$$ Figure 2 ), that embodies many of the visioning suggestions. This concept includes a clinically informative patient list display that supports drilling down to progressively more detailed display of individual patient information. It includes improved quantitative data presentation, the use of color and symbolic coding to present information criticality, symbols to indicate the direction and magnitude of clinically meaningful change, and an integrated approach to CDS.

\section{Discussion}

Our contextually grounded information interviews provided insight into the ways clinicians use information in critical care. These findings correspond with those of Grinspan et al [40] who, in the context of information display design to support care in a pediatric neurology ICU, identified the need to support rapid retrospective review and current status updates. Other studies have focused on information needs at an initial patient encounter or for a patient not previously (or recently) known to the clinician $[21,30,41,55]$. Display design primarily to support an initial patient review leaves out frequent and important information use contexts in critical care. Our findings highlight the need to design systems that will provide fast access to information that has been added to the patient record since the clinician last interacted with that record. Considering an organization of information that separates acute from historical patient data also will help to support these goals. Validating previous work $[20,21,55]$, organization of information by systems or problems also was supported our findings.

Important to critical care clinicians was the presentation of dynamic data that is generated during the patients stay such as vital signs, lab values, and fluid intake and output. This highlights the need for greater attention to issues of presenting data over time to allow clinicians to evaluate trends, whether through tabular or graphic format, and issues of grouping these data so that related information can be easily compared. Our visioning activity suggested that this may be an area where intelligence could be applied, for example, to indicate clinically relevant patient-specific direction and rate of change information. Other approaches to tackling these issues have been evaluated. Bauer, Guerlain, and Brown compared the use of sparklines [26], a form of compact trend graphs, which support the display of multiple trends (eg, 13 variables from a point-of-care blood analyzer) using minimal screen real estate (less than required by a tabular format). Their study suggested an advantage of presentation of data in this format compared to a tabular format for most tasks. The findings of our study support the use of this type of display to address the information needs expressed by our participants. An important caveat is the question of system performance. If such displays take a long time to load and display, clinicians will choose a faster display, even if the format is less desirable. A design proposed by Faiola, Srinivas, and Duke [24] supports a larger scale trend display which provides flexibility to allow clinicians to select the data they wish to simultaneously display (eg, bedside data vital signs, ventilator data, laboratory data). While this solution supports the need to group related information together, our study suggested that clinicians will be unlikely to accept a display format that requires them to choose data to be plotted, at least for typical use. The solution posed by Pickering et al [20] embodies many of the principles indicated by our findings - showing the most recent data first, grouping information by systems, supporting progressive detail. Their design provides trend information by allowing clinicians to, from a display of most recent data points, select an individual variable for a pop-up trend display. However, multiple trends cannot be simultaneously displayed. 
Usability problems associated with dynamic displays, as well as other usability problems were frequently raised in our interviews. Considering the principles used to categorize EMR usability studies by Zahabi, Kaber, and Swangnetr [13] (based on principles from Molich and Nielsen [56]), the bulk of issues identified fell into the categories of "minimizing cognitive load" and "efficient interaction". Because these have been highlighted in other studies $[13,57]$, they are not discussed in detail here; however, we summarized our findings as they relate to critical care into a preliminary set of design guidelines in supplemenatry file Appendix B. Recommendations for improving electronic progress notes, supporting paper-based processes, and task management are also described in supplemenatry file $>$ Appendix B.

Our findings also highlighted the need to support multiple patient views. Critical care clinicians are not always thinking about one patient at a time. They must also balance their time and resources across a group of patients. Visioning also identified the need to integrate CDS into the overall display design. Such an approach would integrate CDS into both single and multiple patient views.

The goals of our study were broad and the coding methods open and flexible. Thus, the direction of data collection, coding, and analysis was influenced by the viewpoint of the investigators. The viewpoint of initial coding was grounded in the discipline of human factors engineering. Validation and visioning incorporated the viewpoint of individuals with expertise as critical care providers and informaticists. Although we collected data in multiple settings, there were differences across settings and it is possible that themes identified here would not apply in other settings. The approach for our visioning activity was not subjected to the same methodological rigor as the eye-tracking data collection and grounded-theory coding activity.

\section{Conclusions}

We conducted retrospective eye-tracking cued interviews toward understanding information use in critical care. Key findings include the need to support new patient review, known patient status review, specific directed information seeking, and multiple patient views. There is a need for improved information organization, simpler and more efficient navigation, and better formatting of dynamic patient data, such as vital signs and laboratory results. Visioning interviews highlighted potential opportunities to improve information presentation for critical care through the use of intelligence and advanced graphical user interface techniques to improve information organization, and prioritization. Some of these ideas may be generalizable to other clinical contexts, particularly acute care settings. It is important that designs require minimal interaction on the part of users to access the information needed. To support this, additional research is necessary to identify and describe clinically meaningful relationships among data and to appropriately define the priority, criticality, and frequency of use of information available to critical care clinicians. Our methods and findings are consistent with recommendations outlined in collaborative work to guide the design of future EHRs [18].

\section{Clinical Relevance}

This research enhances our understanding of the way critical care clinicians use information to support patient care decisions. The results from this study can be used to guide the design of future information display for critical care.

\section{Conflict of Interest}

All authors declare they have no conflicts of interest.

\section{Human Subjects Protections}

The research plan and consent process was approved by the Duke University Health System and Saint Alphonsus Regional Health System Institutional Review Boards ( $\$$ Appendix A for additional human subjects information).

\section{Acknowledgments}

The authors would like to thank the critical care clinicians at Duke University Medical Center and Saint Alphonsus Regional Medical Center for their contributions to this work. We also thank Kathy Gage for her editorial assistance. 


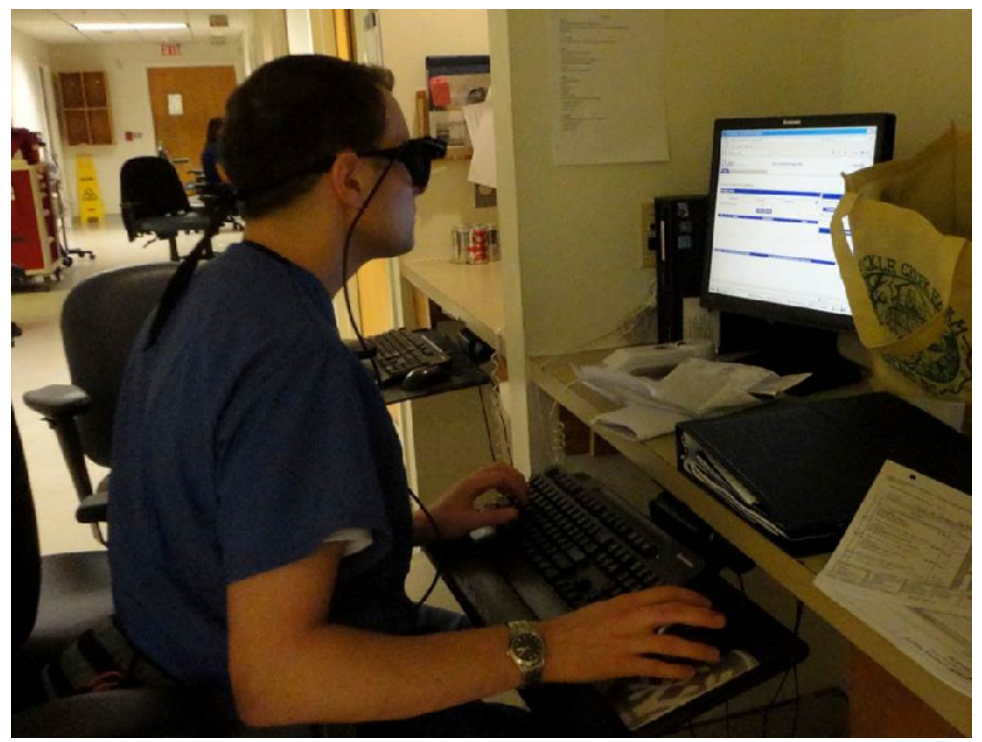

Fig. 1 Provider wearing eye tracker while working. Data was collected in the context of electronic information use and other activities such as rounding, reviewing paper documentation, and working with other clinicians.

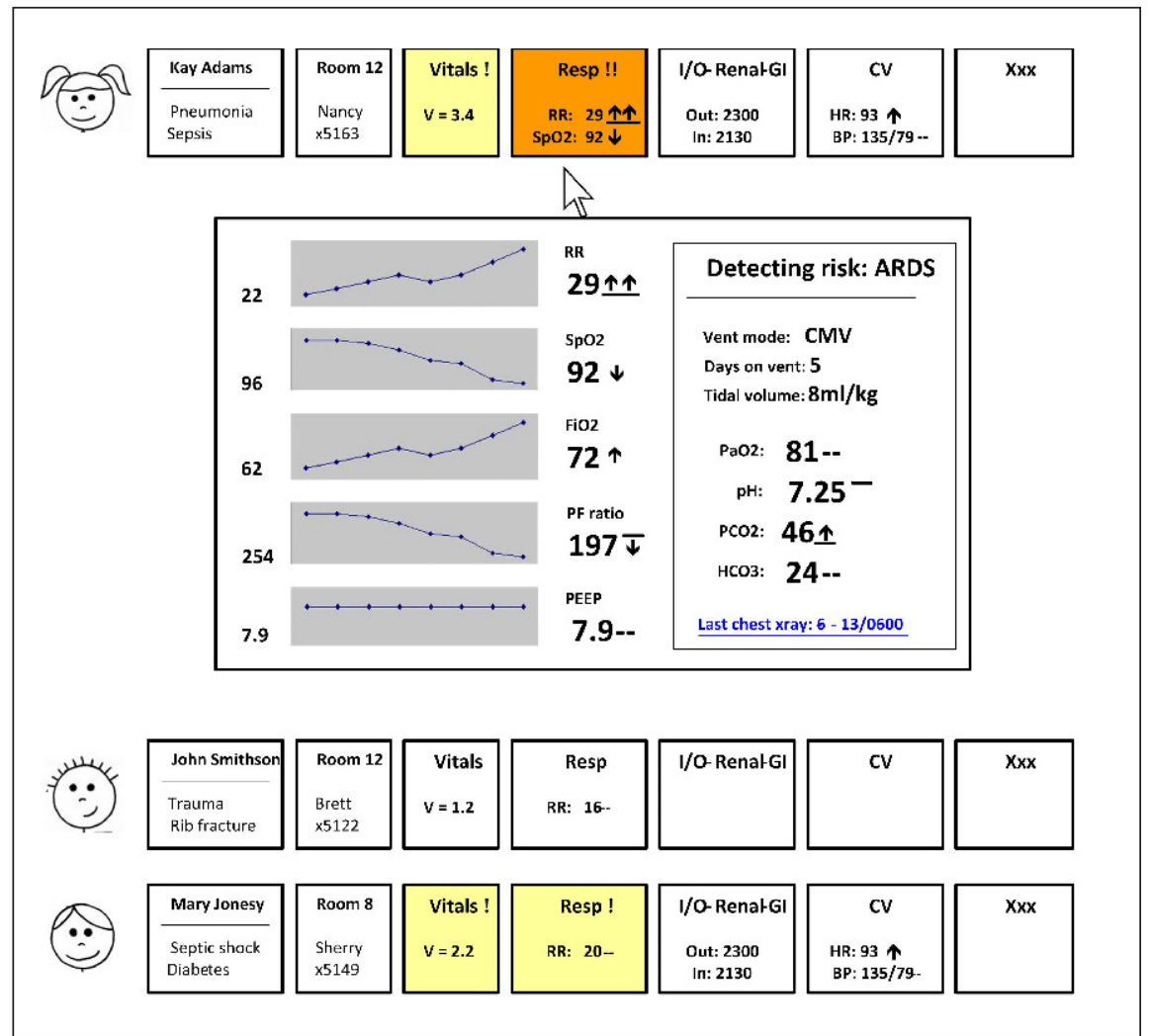

Fig. 2 Example of a more informative patient list display with improved data presentation. This example shows a patient list organized by medical concepts and human body systems. Redundant color and symbol coding highlights status by system. The most recent data for key information are displayed on each patient row with symbolic coding (arrows and bars) to indicate change, rate of change, and deviation from normal range. Progressive detail is accessed by hovering (respiratory details shown), or, for more detail (not shown), selecting a specific system or content grouping. Improved quantitative data presentation is exemplified in the simple graphical plots of related data over time. 
Table 1 Number of eye tracking-based interviews across participant type and care setting

\begin{tabular}{|l|l|l|l|l|}
\hline & $\begin{array}{l}\text { Duke University } \\
\text { SICU }\end{array}$ & $\begin{array}{l}\text { Duke University } \\
\text { NICU }\end{array}$ & SARMC ICU & Total \\
\hline Attending physicians & 4 & 1 & 3 & 8 \\
\hline Residents, fellows, nurse practitioners & 3 & 3 & 2 & 8 \\
\hline Nurses & 0 & 2 & 2 & 4 \\
\hline Total & 7 & 6 & 7 & 20 \\
\hline
\end{tabular}

Table 2 Emerging themes from contextual data interpretation and grounded theory coding

\begin{tabular}{|c|c|}
\hline $\begin{array}{l}\text { Goals and contexts of informa- } \\
\text { tion use }\end{array}$ & $\begin{array}{l}\text { Role is to care for the whole patient with a focus on acute problems } \\
\text { New patient assessment } \\
\text { Known patient status review } \\
\text { Specific directed information seeking } \\
\text { Review and prioritization of multiple patients }\end{array}$ \\
\hline $\begin{array}{l}\text { Emerging themes from grounded } \\
\text { theory coding }\end{array}$ & $\begin{array}{l}\text { Representing dynamic data } \\
\text { What's new } \\
\text { Paper flow sheet reminiscing } \\
\text { Trends } \\
\text { Laboratory data } \\
\text { See related information together } \\
\text { Usability } \\
\text { Speed of information retrieval } \\
\text { System and navigation complexity } \\
\text { Display clutter and hidden information } \\
\text { Pop-ups } \\
\text { Cross-system integration } \\
\text { Processes for managing information } \\
\text { Summary information for team work } \\
\text { Summary information for personal use } \\
\text { Progress notes } \\
\text { Task tracking }\end{array}$ \\
\hline
\end{tabular}




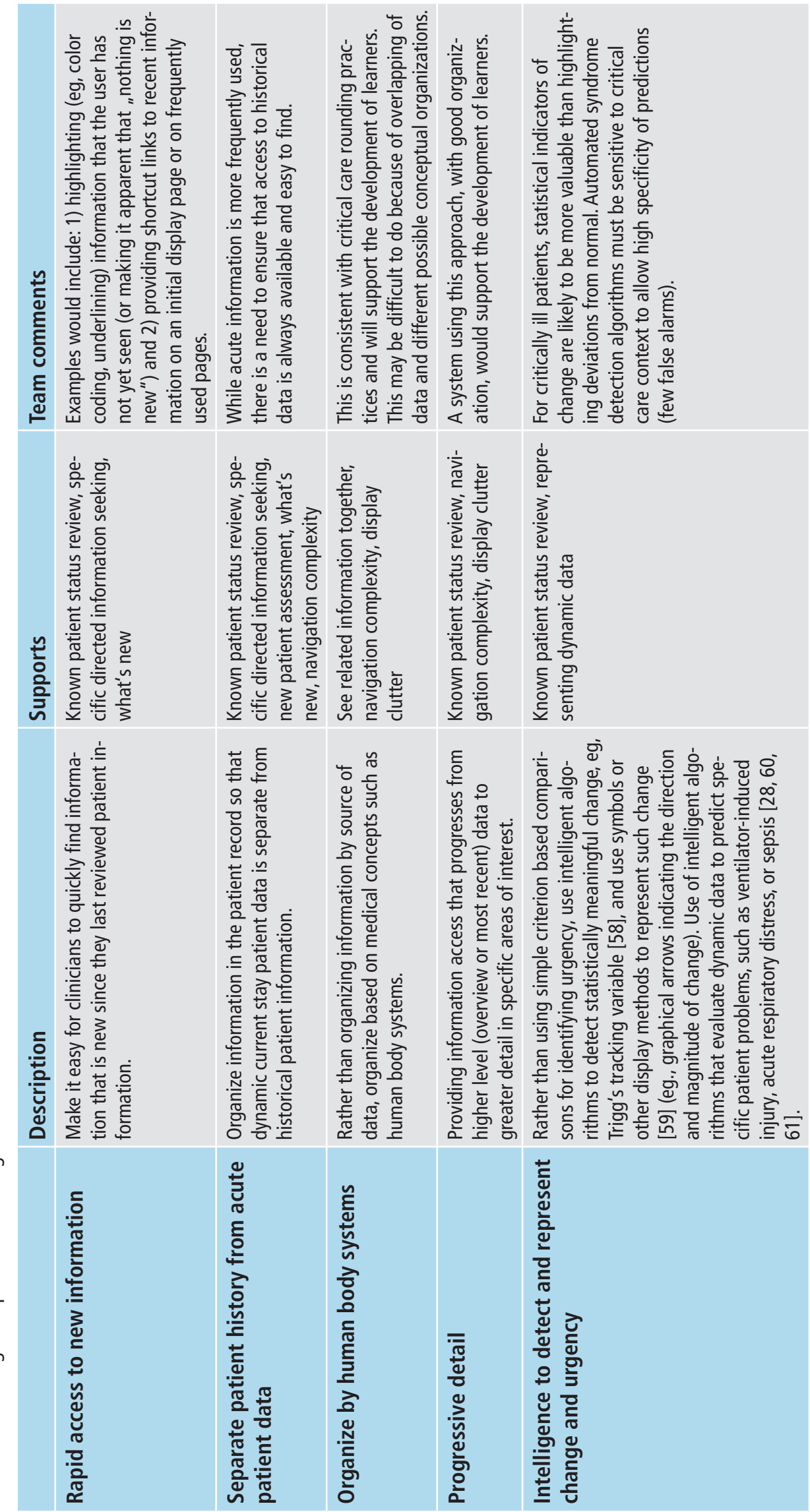



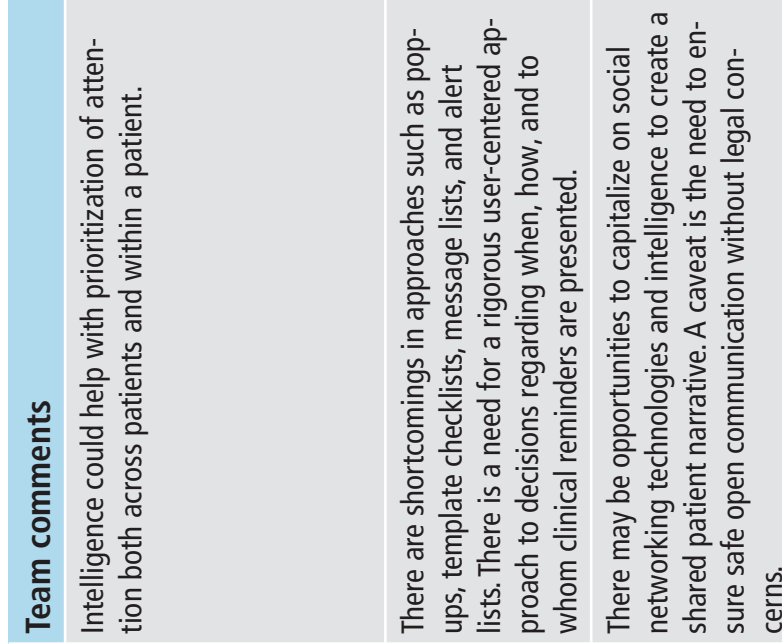

는

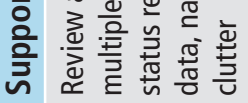

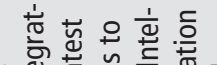

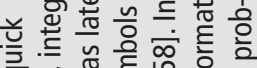

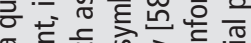

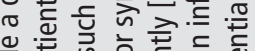

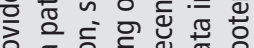

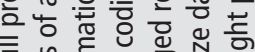

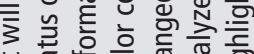

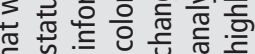
는 윤 층 른 은 을 언

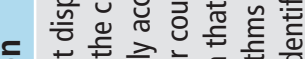
흥

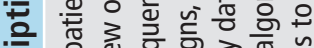

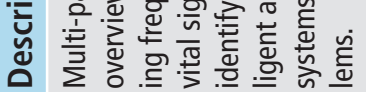
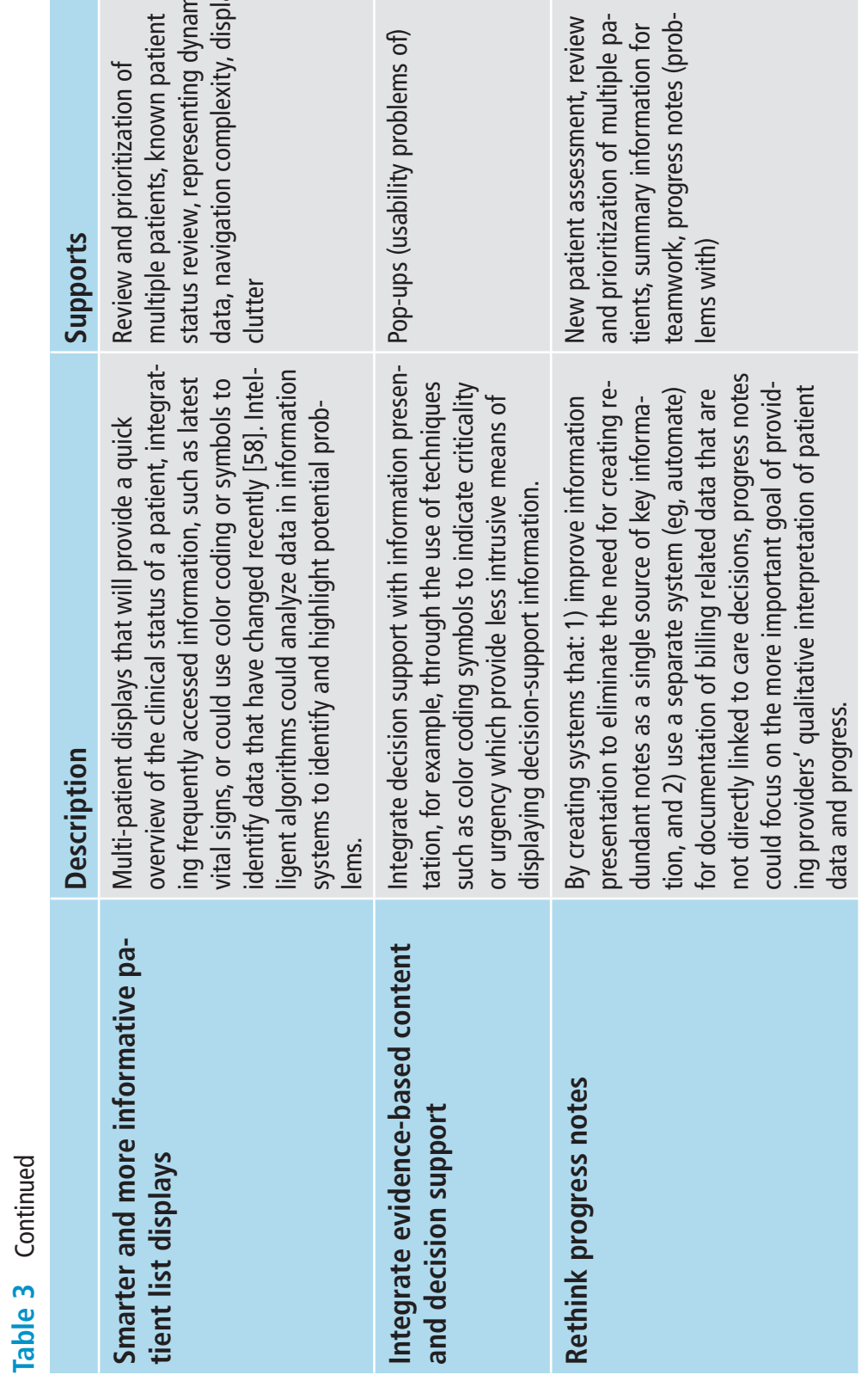

(C) Schattauer 2016 Melanie C. Wright et al.: Toward designing information display to support critical care 


\section{References}

1. Newman-Toker D, Pronovost P. Diagnostic Errors--The Next Frontier for Patient Safety. JAMA: The Journal of the American Medical Association 2009; 301(10): 1060-1062.

2. Leape LL, Berwick D, Bates D. Counting deaths due to medical errors. JAMA 2002; 288(19): 2405.

3. Donchin Y, Gopher D, Olin M, Badihi Y, Biesky M, Sprung CL, Pizov R, Cotev S. A look into the nature and causes of human errors in the intensive care unit. Crit Care Med 1995; 23(2): 294-300.

4. Bracco D, Favre J, Bissonnette B, Wasserfallen J, Revelly J, Ravussin P, Chiolero R. Human errors in a multidisciplinary intensive care unit: a 1-year prospective study. Intensive Care Medicine 2001; 27(1): 137-145.

5. Forster A, Kyeremanteng K, Hooper J, Shojania K, Van Walraven C. The impact of adverse events in the intensive care unit on hospital mortality and length of stay. BMC health services research 2008; 8(1): 259.

6. Fackler J, Watts C, Grome A, Miller T, Crandall B, Pronovost P. Critical care physician cognitive task analysis: an exploratory study. Crit Care 2009; 13(2): R33.

7. Ahmed AH, Giri J, Kashyap R, Singh B, Dong Y, Kilickaya O, Erwin PJ, Murad MH, Pickering BW. Outcome of adverse events and medical errors in the intensive care unit: a systematic review and meta-analysis. Am J Med Qual 2015; 30(1): 23-30.

8. Meeks DW, Smith MW, Taylor L, Sittig DF, Scott JM, Singh H. An analysis of electronic health record-related patient safety concerns. J Am Med Inform Assoc 2014; 21(6): 1053-1059.

9. Zhang J, Walji MF. TURF: Toward a unified framework of EHR usability. J Biomed Inform. 2011 Dec;44(6):1056-67.

10.Schumacher RM, Berkowitz L, Abramson P, Liebovitz D. Electronic Health Records: Physicians Perspective on Usability. In: Proceedings of the Human Factors and Ergonomics Society 54th Annual Meeting. Santa Monica CA: Human Factors and Ergonomics Society; 2010.

11. Gans D, Kralewski J, Hammons T, Dowd B. Medical groups' adoption of electronic health records and information systems. Health Aff (Millwood) 2005; 24(5): 1323-1333.

12. Armijo D, McDonnell C, Werner K. Electronic Health Record Usability: Interface Design Considerations. Rockville, MD; 2009. Report No:

13.Zahabi M, Kaber DB, Swangnetr M. Usability and Safety in Electronic Medical Records Interface Design: A Review of Recent Literature and Guideline Formulation. Hum Factors 2015; 57(5): 805-834.

14.Zhou YY, Kanter MH, Wang JJ, Garrido T. Improved quality at Kaiser Permanente through e-mail between physicians and patients. Health Aff (Millwood) 2010; 29(7): 1370-1375.

15. Russ A, Saleem J, Justice C, Hagg H, Woodbridge P, Doebbeling B. Healthcare Workers' Perceptions of Information in the Electronic Health Record. Proceedings of the Human Factors and Ergonomics Society 53rd Annual Meeting. Santa Monica CA: Human Factors and Ergonomics Society; 2009.

16. Weir CR, Nebeker JR. Critical issues in an electronic documentation system. AMIA Annual Symposium proceedings / AMIA Symposium AMIA Symposium 2007: 786-790.

17. Jaspers MW, Peute LW, Lauteslager A, Bakker PJ. Pre-post evaluation of physicians' satisfaction with a redesigned electronic medical record system. Stud Health Technol Inform 2008; 136: 303-308.Payne TH, Corley S, Cullen TA, Gandhi TK, Harrington L, Kuperman GJ, Mattison JE, McCallie DP, McDonald CJ, Tang PC, Tierney WM, Weaver C, Weir CR, Zaroukian MH. Report of the AMIA EHR囚2020 Task Force on the status and future direction of EHRs. J Am Med Inform Assoc; 22(5): 1102-1110.

18. Payne TH, Corley S, Cullen TA, Gandhi TK, Harrington L, Kuperman GJ, Mattison JE, McCallie DP, McDonald CJ, Tang PC, Tierney WM, Weaver C, Weir CR, Zaroukian MH. Report of the AMIA EHR冈2020 Task Force on the status and future direction of EHRs. J Am Med Inform Assoc 2015; 22(5): 1102-1110.

19. Carayon P, Wetterneck TB, Alyousef B, Brown RL, Cartmill RS, McGuire K, Hoonakker PL, Slagle J, Van Roy KS, Walker JM, Weinger MB, Xie A, Wood KE. Impact of electronic health record technology on the work and workflow of physicians in the intensive care unit. International journal of medical informatics 2015; 84(8): 578-594.

20.Pickering BW, Dong Y, Ahmed A, Giri J, Kilickaya O, Gupta A, Gajic O, Herasevich V. The implementation of clinician designed, human-centered electronic medical record viewer in the intensive care unit: a pilot step-wedge cluster randomized trial. International journal of medical informatics 2015; 84(5): 299-307.

21.Zeng Q, Cimino JJ, Zou KH. Providing concept-oriented views for clinical data using a knowledge-based system: an evaluation. J Am Med Inform Assoc 2002; 9(3): 294-305.

22. Gorges M, Kuck K, Koch SH, Agutter J, Westenskow DR. A far-view intensive care unit monitoring display enables faster triage. Dimensions of critical care nursing: DCCN 2011; 30(4): 206-217. 
23. Koch SH, Weir C, Westenskow D, Gondan M, Agutter J, Haar M, Liu D, Gorges M, Staggers N. Evaluation of the effect of information integration in displays for ICU nurses on situation awareness and task completion time: A prospective randomized controlled study. Int J Med Inform 2013; 82(8): 665-675.

24. Faiola A, Srinivas P, Duke J. Supporting Clinical Cognition: A Human-Centered Approach to a Novel ICU Information Visualization Dashboard. AMIA Annu Symp Proc 2015; 2015: 560-569.

25. Holmes C, Brown M, Hilaire DS, Wright A. Healthcare provider attitudes towards the problem list in an electronic health record: a mixed-methods qualitative study. BMC medical informatics and decision making 2012; 12: 127.

26. Bauer DT, Guerlain S, Brown PJ. The design and evaluation of a graphical display for laboratory data. J Am Med Inform Assoc 2010; 17(4): 416-424.

27. Evans RS, Larsen RA, Burke JP, Gardner RM, Meier FA, Jacobson JA, Conti MT, Jacobson JT, Hulse RK. Computer surveillance of hospital-acquired infections and antibiotic use. JAMA 1986; 256(8): 1007-1011.

28. Herasevich V, Tsapenko M, Kojicic M, Ahmed A, Kashyap R, Venkata C, Shahjehan K, Thakur SJ, Pickering BW, Zhang J, Hubmayr RD, Gajic O. Limiting ventilator-induced lung injury through individual electronic medical record surveillance. Crit Care Med 2011; 39(1): 34-39.

29. Kannampallil TG, Jones LK, Patel VL, Buchman TG, Franklin A. Comparing the information seeking strategies of residents, nurse practitioners, and physician assistants in critical care settings. J Am Med Inform Assoc 2014; 21(e2): e249-e256.

30. Pickering BW, Gajic O, Ahmed A, Herasevich V, Keegan MT. Data Utilization for Medical Decision Making at the Time of Patient Admission to ICU*. Crit Care Med 2013; 41(6): 1502-1510.

31. Saleem JJ, Plew WR, Speir RC, Herout J, Wilck NR, Ryan DM, Cullen TA, Scott JM, Beene MS, Phillips T. Understanding barriers and facilitators to the use of Clinical Information Systems for intensive care units and Anesthesia Record Keeping: A rapid ethnography. Int J Med Inform 2015; 84(7): 500-511.

32. Yusof MM. A case study evaluation of a Critical Care Information System adoption using the socio-technical and fit approach. Int J Med Inform 2015; 84(7): 486-499.

33. Endsley MR, Bolte B, Jones DG. Designing for Situation Awareness: An Approach to Human-Centered Design. London: Taylor and Francis; 2003.

34. Holtzblatt K, Wendell J, Wood S. Rapid Contextual Design. San Francisco: Elsevier, Inc.; 2005.

35. Vicente KJ. Ecological interface design: progress and challenges. Hum Factors 2002; 44(1): 62-78.

36. Vicente KJ, Rasmussen J. Ecological interface design: Theoretical foundations. IEEE Transactions on Systems, Man, and Cybernetics 1992; 22(4): 589-606.

37. Burns CM, Hajdukiewicz JR. Ecological Interface Design. Boca Raton FL: CRC Press; 2004.

38. Benard H, Killworth P, Kronenfeld D, Sailer L. The problem of informant accuracy: the validity of retrospective data. Annual Review of Anthropology 1984; 13: 495-517.

39. Klein D. When to ignore what people say. Ergonomics in Design. 2006;Winter.

40. Grinspan Z, Eldar Y, Gopher D, Gottlieb A, Lammfromm R, Mangat H, Peleg N, Pon S, Rozenberg I, Schiff N, Stark D, Yan P, Pratt H, Kosofsky B. Findings from an International Working Group. Applied Clinical Informatics 2016; 7(2): 380-398.

41.Doberne JW, He Z, Mohan V, Gold JA, Marquard J, Chiang MF. Using High-Fidelity Simulation and Eye Tracking to Characterize EHR Workflow Patterns among Hospital Physicians. AMIA Annu Symp Proc 2015; 2015: 1881-1889.

42.Henneman E, Marquard J, Fisher D, Gawlinski A. Eye tracking: A novel approach for evaluating and improving the safety of healthcare processes. Simulation in Healthcare. In press.

43. Nelson SD, LaFleur J, Del Fiol G, Evans RS, Weir CR. Reading and Writing: Qualitative Analysis of Pharmacists' Use of the EHR when Preparing for Team Rounds. AMIA Annu Symp Proc 2015; 2015: 943-952.

44. Seagull FJ, Xiao Y. Using eye-tracking video data to augment knowledge elicitation in cognitive task analysis. Proceedings of the Human Factors and Ergonomics Society 45th Annual Meeting. Santa Monica, CA: Human Factors and Ergonomics Society; 2001; 400-403.

45. Tien T, Pucher PH, Sodergren MH, Sriskandarajah K, Yang GZ, Darzi A. Eye tracking for skills assessment and training: a systematic review. J Surg Res 2014; 191(1): 169-178.

46. Wright M, Dunbar S, Moretti E, Schroeder R, Taekman J, Segall N, editors. Eye-Tracking and Retrospective Verbal Protocol to Support Information Systems Design. Human Factors and Ergonomics Society Health Care Symposium; 2013; Baltimore, MD. Human Factors and Ergonomics Society.

47.Duchowski A. Eye Tracking Methodology: Theory and Practice. 2nd ed. London: Springer; 2007.

48. Henneman E, Marquard J, Fisher D, Gawlinski A. Eye tracking: A novel approach for evaluating and improving the safety of healthcare processes. Simulation in Healthcare. Under review.

49. Poole A, Ball LJ. Eye-trackng in HCI and usability research. Encyclopedia of Human Computer Interaction 2006; 1: 211-219. 
50. Kushniruk AW, Patel VL, Cimino JJ. Usability testing in medical informatics: cognitive approaches to evaluation of information systems and user interfaces. Proceedings : a conference of the American Medical Informatics Association / AMIA Annual Fall Symposium AMIA Fall Symposium. 1997; Jan 1:218-222.

51. Charmaz K. Constructing Grounded Theory. Thousand Oaks, CA: Sage Publications Inc.; 2006.

52. Beyer H, Holtzblatt K. Contextual Design: Defining Customer-Centered Systems. San Francisco CA: Morgan Kaufmann; 1977.

53.Steinhauser KE. Qualitative Research Methods: An Overview. Clinical Research Training Program: Health Services Research: Duke University Medical Center; 2009.

54. Mays N, Pope C. Qualitative research: Observational methods in health care settings. BMJ. 1995 Jul 15;311(6998):182-4.

55.Zheng K, Padman R, Johnson MP, Diamond HS. An interface-driven analysis of user interactions with an electronic health records system. J Am Med Inform Assoc 2009; 16(2): 228-237.

56. Molich R, Nielsen J. Improving a human-computer dialogue. Communications of the ACM. 1990;33:338-48.

57. Bates DW, Kuperman GJ, Wang S, Gandhi T, Kittler A, Volk L, Spurr C, Khorasani R, Tanasijevic M, Middleton B. Ten commandments for effective clinical decision support: making the practice of evidencebased medicine a reality. J Am Med Inform Assoc 2003; 10(6): 523-530.

58. Kennedy RR, Merry AF. The effect of a graphical interpretation of a statistic trend indicator (Trigg's Tracking Variable) on the detection of simulated changes. Anaesthesia and intensive care 2011; 39(5): 881-886.

59. Tappan JM, Daniels J, Slavin B, Lim J, Brant R, Ansermino JM. Visual cueing with context relevant information for reducing change blindness. J Clin Monit Comput 2009; 23(4): 223-232.

60.Herasevich V, Kor DJ, Subramanian A, Pickering BW. Connecting the dots: rule-based decision support systems in the modern EMR era. Journal of clinical monitoring and computing 2013; 27(4): 443-448.

61.Lynn LA, Curry JP. Patterns of unexpected in-hospital deaths: a root cause analysis. Patient safety in surgery $2011 ; 5(1): 3$. 